REVUE HYBRIDE DE L'ÉDUCATION

\title{
Création du groupe « Stratégies Plus »
}

\author{
Auteurs \\ Stéphane Maltais, École l'Odyssée, Commission scolaire des Affluents, \\ Canada, \\ stephane.maltais@csda.ca \\ Yves Daigneault, École l'Odyssée, Commission scolaire des Affluents, \\ Canada
}




\section{REVUE HYBRIDE DE L’ÉDUCATION}

\section{Résumé}

Créé en 2014-2015 à l'école secondaire de l'Odyssée, le groupe "Stratégies Plus » réunit divers intervenants du milieu scolaire afin de soutenir les élèves qui ont des difficultés en mathématiques et en français. Animé par la volonté de « faire autrement », il privilégie le coenseignement et la concertation entre les participants. Sa mise en place a entrainé de nombreux changements dans l'organisation et les pratiques scolaires. Après quatre années d'existence, les bénéfices pour les élèves sont mesurables et le groupe "Stratégie Plus » a acquis une bonne réputation auprès des autres enseignants, des élèves et des parents.

Mots-clés : stratégies plus, coenseignement, concertation, organisation du travail, planification, gestion du temps 


\section{$\&$}

\section{REVUE HYBRIDE DE L'ÉDUCATION}

\section{Mise en contexte}

Le groupe "Stratégies Plus " a été créé à la suite d'un constat de la direction de l'École secondaire de l'Odyssée qui souhaitait rehausser le taux de diplomation de ses élèves et amenuiser le taux de décrochage scolaire au sein de l'établissement. Parmi les moyens pour améliorer ces deux aspects, l'idée de créer un groupe particulier qui ciblerait des élèves de troisième secondaire ayant des problématiques dans les matières de base, notamment en mathématiques et en français a fait son chemin.

En collaboration avec la Commission scolaire des Affluents ainsi qu'une équipe d'intervenants et d'enseignants volontaires, le groupe en question a vu le jour en 2014-2015. Une expression revenait lors de l'établissement des objectifs pédagogiques de ce groupe: «faire différemment ». Le mandat pour cette cohorte d'élèves que l'on a baptisé, par la suite, groupe «Stratégies Plus », était de prioriser une meilleure compréhension en lecture en impliquant l'enseignant de français en mathématiques, entre autres, et de favoriser une compréhension accrue des notions mathématiques en ajoutant deux périodes à la grille-horaire. C'est ainsi que le modèle de coenseignement mathématiques-français a été instauré.

De plus, pour s'assurer d'être en lien direct avec l'idée de «faire différemment », la commission scolaire a injecté des sommes afin de dégager la conseillère en orientation à raison d'une journée par semaine, et ainsi, répondre aux besoins des élèves qui perdaient deux périodes par cycle pour le cours de projet personnel d'orientation (PPO). En agissant ainsi, on s'assurait du même coup de garder l'élève directement impliqué dans son apprentissage et, par le fait même, d'optimiser la motivation face à son parcours scolaire en lui donnant un sens. II va sans dire que la direction de l'école a aussi cherché à recruter une équipe d'enseignants paritaire hommes-femmes qui étaient motivés à faire partie de ce qui allait devenir un beau projet. Le fondement d'un tel groupe repose en très grande partie sur le développement du lien professeur-élèves pour faire vivre à ces derniers de petits succès à l'aide de la théorie des petits pas. Les élèves sélectionnés provenaient de groupes de «régulier» et de classes ressources, ou étaient des élèves doubleurs pour qui la réussite scolaire était peu présente dans leur parcours. Le groupe "Stratégies Plus 》 avait pour but de leur donner la chance de vivre des succès et de les engager dans leur cheminement scolaire.

\section{Un projet en constante évolution}

Actuellement, le groupe "Stratégies Plus » en est à sa quatrième édition à l'École secondaire de l'Odyssée. Il est bien établi dans son milieu. II est évident que plusieurs ajustements ont dû être nécessaires pour maximiser l'efficacité du groupe d'intervenants face à ce type d'élèves. Nombreux ont été les questionnements : quelle clientèle prioriser dans ce 


\section{$\&$}

\section{REVUE HYBRIDE DE L'ÉDUCATION}

groupe? Comment faire différemment? Quels modèles de coenseignement utiliser? Comment favoriser un temps d'étude optimal pour mener les élèves à la réussite ? Bref, il y a eu évolution avec le temps et les pratiques sont en perpétuel avancement. Par exemple, le coenseignement de l'enseignant de français dans le cours de mathématiques, qui à l'époque cherchait sa raison d'être, a trouvé son utilité et a produit de bons résultats. Le temps d'étude à l'horaire est aussi un exemple qui a mené à de belles réussites. Année après année, la pratique a progressé et le mode de fonctionnement s'est circonscrit au point de devenir un modèle de référence dans notre commission scolaire.

\section{Composition du groupe}

Comme mentionné ci-dessus, la clientèle recherchée pour le groupe "Stratégies Plus " a généré de nombreux questionnements et a donné lieu à plusieurs discussions. L'enjeu était de déterminer le type d'élèves à prioriser dans ce regroupement. II est évident que les étudiants formant cette cohorte éprouvent des difficultés scolaires ou vivent des problématiques diverses. Ces problématiques font de ces élèves des candidats potentiels au décrochage scolaire. En ce sens, l'équipe d'intervenants (enseignants de deuxième secondaire, la technicienne en éducation spécialisée et la direction) qui sélectionne les élèves qui composent le groupe "Stratégies Plus » tente de rassembler des élèves qui ont un certain intérêt face à leur réussite, qui sont prêts à fournir un effort et qui ont de nombreuses lacunes en mathématiques et en français. Depuis l'an un, les groupes varient entre 22 et 26 élèves. Tous les parents des futurs protagonistes sont consultés et informés par téléphone à la fin de l'année de leur premier cycle. Comme c'est un groupe maintenant établi dans son milieu, la réputation de ce dernier a évolué avec le temps. En effet, les doutes ou la crainte du jugement des autres se sont dissipés avec le temps. Les élèves et leurs parents ont été rassurés, voire encouragés lorsqu'ils ont expérimenté le dispositif. Le choix éclairé des élèves sélectionnés et l'adéquation des services offerts en fonction de leur réalité ont permis de créer une réputation positive autour du dispositif.

\section{Comment se vit le groupe «Stratégies Plus » ?}

Il est d'abord important de rappeler que le groupe «Stratégies Plus » a un curriculum particulier. II faut savoir que le contenu de toutes les matières est le même que les autres élèves de troisième secondaire. Toutefois, comme il a été relevé plus tôt, le cours de projet personnel d'orientation (PPO) se donne en deux périodes par cycle au lieu de quatre, afin de laisser place à deux périodes de plus en mathématiques et ainsi faciliter la tâche des élèves ayant plus de difficulté dans cette matière. II faut rappeler que la conseillère en orientation est en soutien aux étudiants afin de pallier le manque en projet personnel d'orientation (PPO). La modification d'horaire, soit huit périodes par cycle en mathématiques, permet vraiment à l'enseignant de cette matière de rétablir l'écart quant aux 


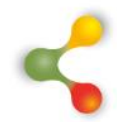

\section{REVUE HYBRIDE DE L'ÉDUCATION}

retards importants de cette clientèle. Ces 40 périodes additionnelles de mathématiques favorisent un apprentissage plus adéquat, des explications plus approfondies et une mise en application des notions par le biais d'exercices plus fréquents. Ajoutons que les élèves du groupe ont aussi la chance d'assister à de nombreuses récupérations en mathématiques après l'école. Du même coup, les deux périodes de mathématiques ajoutées au calendrier peuvent servir, dans certains cas, à travailler des thématiques jugées importantes par l'équipe. Les thématiques en question peuvent être transmises par des intervenants différents. II a, par exemple, été question d'hygiène de vie, de planification d'études, d'un programme appelé "Déstresse progresse ", de choix de cours et de types d'apprenants, au cours des dernières années. Ces périodes servant surtout aux mathématiques permettent tout de même une certaine flexibilité à I'horaire.

De plus, l'enseignant de mathématiques bénéficie d'une libération de six périodes par cycle en enseignement ressource, spécifiquement pour ce groupe. II va sans dire que son implication a une valeur inestimable, car l'équipe entière (élèves, enseignants, intervenants et direction) se sent pleinement appuyée et il est beaucoup plus facile de mettre des pratiques concrètes en place avec un enseignant disponible six cours par cycle. Avec ce dégagement d'horaire, celui-ci accompagne souvent les autres professeurs dans leur cours respectif et tout le monde en est gagnant.

La grille-horaire du groupe "Stratégies Plus» favorise donc grandement le coenseignement. Comme mentionné auparavant, l'enseignant de français se rend en mathématiques deux cours par cycle. Ce temps lui est reconnu dans sa tâche à 26 périodes par neuf jours au lieu de 24 , et l'expérience a permis de mettre en place des pratiques très probantes. Le modèle de coenseignement varie selon les apprentissages. Par exemple, il n'est pas rare de voir le professeur de français exécuter un problème mathématique au tableau, devant le groupe, alors que les élèves le guident totalement. L'enseignant de français n'ayant pas les compétences pour la réalisation du problème en question, leur appui est donc nécessaire et indispensable pour la démarche. Tous ensemble, ils trouvent la solution. Cette pratique a permis de constater que le fait d'aller au rythme des élèves aidait grandement leur compréhension. De plus, verbaliser les interventions à voix haute permettait d'aider les plus faibles à pallier les problématiques en cours d'apprentissage. Enfin, voir un enseignant placé en position vulnérable n'est pas sans créer une atmosphère conviviale laissant place à des moments humoristiques, ce que les élèves apprécient grandement.

Différentes stratégies d'enseignement peuvent aussi être mises en $œ u v r e$. Parmi celles expérimentées, en voici quelques-unes qui se sont avérées fructueuses. Diviser le groupe en deux peut permettre à l'enseignant de mathématiques de faire de la remédiation avec un petit groupe d'élèves plus en difficultés. Lorsque c'est possible, les enseignants 


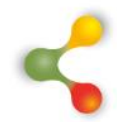

\section{REVUE HYBRIDE DE L'ÉDUCATION}

utilisent deux locaux pour que les élèves qui travaillent ne soient pas dérangés par ceux qui ont une activité de remédiation.

Le travail en atelier constitue aussi une stratégie pédagogique intéressante. II peut s'agir de diviser le groupe en équipes d'experts. Ces derniers s'expliquent à tour de rôle les notions vues dans une séquence ponctuelle précise et cela facilite les apprentissages. Les deux enseignants sont donc en circulation dans la classe afin d'intervenir et d'échanger avec les équipes. L'élève est réellement en action, ce qui lui permet de sortir de la routine. Le fait d'être deux intervenants assure donc que le travail soit bien fait et réalisé dans le temps imparti.

Enfin, des formules qui permettent de vérifier rapidement la compréhension des élèves ont aussi été expérimentées. À titre d'exemple, attribuer un petit tableau blanc aux élèves afin qu'ils écrivent leur réponse permet d'un seul coup à l'enseignant de repérer ceux qui ont compris et ceux pour qui la notion reste à travailler. La régulation se fait alors par l'enseignant qui circule, ou peut donner lieu à une capsule de rappel pour consolider les contenus à apprendre.

Parmi les belles réussites du groupe "Stratégies Plus », la mise en place de périodes d'étude à l'horaire mérite d'être soulignée. Depuis trois ans, tous les mardis, une période $\left(2^{\mathrm{e}}\right.$ ou $\left.3^{\mathrm{e}}\right)$ est consacrée à l'étude. L'instauration de ce temps pédagogique demande de la flexibilité et des compromis de la part de l'équipe d'enseignants. Au total, ce sont 30 périodes, réparties sur l'année scolaire, qui sont utilisées pour offrir ces temps d'étude. Chaque discipline accepte de consacrer des périodes à l'horaire pour cette activité. Ce temps à l'horaire favorise particulièrement le coenseignement. En effet, comme les périodes se passent dans une discipline en particulier, le coenseignant assure ainsi le bon fonctionnement. Par exemple, si le jour 2, à la deuxième période, le groupe est en français, l'enseignant de français sera en classe comme à l'habitude et sera accompagné d'un collègue. Ce coenseignement est déterminé de la façon suivante : chaque professeur a environ quatre périodes à donner dans l'année pour ce temps d'étude. Un calendrier pour l'année entière sera complété au mois de septembre par l'équipe d'enseignants. Chacun choisit les périodes où il se rendra disponible. De même, le calendrier des périodes à l'étude et des contenus à voir est déterminé par l'équipe de professeurs lors d'une rencontre qui se tient tous les mois. Ainsi, s'il y a un examen d'histoire pour le groupe, le jeudi, la période d'étude du mardi précédent portera sur cette discipline. Les élèves apprécient vraiment ce temps précieux qui leur est consacré pour favoriser leur réussite. S'il n'y a pas d'examen, il se peut qu'une période reste inchangée, mais le coenseignement se fait tout de même pendant la période. II est aussi possible que ce cours serve à une matière qui a accumulé du retard. Bref, ce temps à l'horaire a de nombreuses utilités et amène une certaine flexibilité pour le groupe «Stratégies Plus ». 


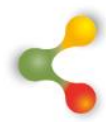

\section{REVUE HYBRIDE DE L'ÉDUCATION}

II est évident qu'un temps est reconnu dans la tâche des enseignants pour toutes les réunions servant à préparer ces périodes d'étude et rencontres qui se font après l'école. Comme ce temps d'étude a été placé à l'horaire en début d'année, il n'est pas rare de voir deux enseignants qui n'ont rien à voir avec la matière étudiée le mardi matin, intervenir avec les élèves. Cette réalité laisse place à des moments uniques, inespérés et cocasses que le groupe apprécie réellement. En effet, les élèves sont parfois témoins de la préparation d'examens de mathématiques qui implique l'enseignant de la discipline et celui d'éducation physique, qui propose son aide spontanément. II s'en suit une véritable collaboration dont les élèves aiment être témoins. En résumé, ce temps à l'horaire est un modèle gagnant qui favorise le lien entre collègues ainsi qu'entre professeurs et élèves, contribuant significativement à l'engagement et à l'apprentissage des élèves.

\section{Parcours des ex-participants}

Lorsqu'un projet de la sorte est mis en place dans un milieu, il est toujours intéressant d'assurer un suivi. Que deviennent les élèves qui sont passés par le groupe "Stratégies Plus »? Voilà une question intéressante à laquelle il est plutôt difficile de répondre. Malgré la complexité de cette tâche, il a été tout de même possible de suivre, en partie, le parcours des " anciens » grâce à la collaboration de celle qui a accompagné ces derniers pendant leur parcours scolaire, soit la conseillère en orientation. Comme l'un des buts était de maintenir les participants actifs dans le réseau scolaire, les conclusions du suivi sont extrêmement positives. De toutes les cohortes des groupes "Stratégies Plus ", seuls cinq élèves à ce jour ne sont plus actifs dans le système scolaire public québécois ou n'ont pas obtenu de diplôme reconnu. Ces derniers proviennent des deux premières cohortes passées dans le groupe. Sont-ils sur le marché du travail, en formation privée, en attente de réintégration scolaire ou à l'extérieur de la province? Impossible de le savoir. Un fait reste assuré, la rétention des élèves qui sont passés par le groupe "Stratégies Plus " est très élevée. Mis à part ces cinq élèves non diplômés, tous les autres ex-participants du groupe sont diplômés ou toujours actifs dans le réseau scolaire public québécois. Sans ce passage, il est évidemment impossible de savoir ce que ces jeunes seraient devenus. Nous sommes fiers de contribuer à la mobilisation et à la persévérance de ces derniers. L'objectif de «faire autrement " s'avère bénéfique et justifie les efforts conjugués que nous déployons pour contribuer à l'optimisation du potentiel de nos élèves.

\section{Rétroaction des anciens élèves et des parents}

Comme il a été mentionné auparavant, le groupe "Stratégies Plus » en est à sa quatrième édition. Il est évident qu'avec les années, ce groupe a grandement évolué. Est-il à son apogée ? Sûrement pas, mais afin de valider le modèle en question, il est toujours intéressant de recueillir des témoignages de la part des participants (parents et élèves) qui ont pris 


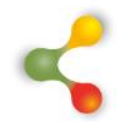

\section{REVUE HYBRIDE DE L'ÉDUCATION}

part à ce groupe. En voici quelques-uns: Allyson insiste sur l'esprit «familial » du groupe : «Au début, je ne voulais absolument pas être dans ce groupe-là, j'ai vraiment fini par m'attacher aux autres. On était comme une grande famille. En plus, j'étais moins gênée et plus à l'aise avec tout le monde ». Elle note l'union qui existait dans ce groupe, ce qu'elle n'a malheureusement pas relevé en quatrième secondaire.

Pour sa part, Joanna mentionne qu'elle aurait souhaité poursuivre l'expérience en quatrième et cinquième secondaires. Kelly va plus loin : "Cette année-là a littéralement changé ma vision de l'école. On a eu du « fun » en classe et on a développé de belles relations qui se poursuivent », mentionne-t-elle. De nombreux élèves évoquent les liens qui se sont tissés dans ce groupe.

De plus, la grande disponibilité des enseignants est un facteur qui a été relevé. David a même mentionné : "Pour une rare fois, les professeurs ne criaient pas dans les cours». Pour Philippe, le groupe "Stratégies Plus » a été "sa plus belle année à vie ". Même si, sans ce passage, il aurait terminé son diplôme d'études secondaires, Philippe a vraiment apprécié la dynamique du groupe et ça lui a permis d'avancer à un rythme qui lui convenait réellement. Doriane va même jusqu'à dire : « Je ne suis pas sûre que je serais en secondaire cinq sans "Stratégies »". Cette jeune fille peu volubile en classe, mentionne garder des souvenirs mémorables de son année de troisième secondaire.

Geneviève qui était de la première cohorte en «Stratégies Plus » a relevé ce jugement des autres à l'égard de ce groupe, ce qui ne lui plaisait pas du tout au départ. Elle ajoute toutefois que ce jugement a vite fait place à de la jalousie au cours des années suivantes, compte tenu des commentaires véhiculés à l'école à l'égard de ce groupe qui vivait des moments un peu différents des autres.

Les commentaires recueillis lors des réunions de parents du début d'année sont aussi fréquents et positifs. Même s'il reste des parents qui n'ont encore aucune idée de ce qu'est le groupe "Stratégies Plus", nombreux sont ceux qui en ont entendu parler positivement. II y en a même certains qui ont avoué souhaiter que leur enfant y soit "sélectionné ». La mère d'un élève du groupe a mentionné qu'elle n'avait encore jamais vu son garçon aussi positif en début d'année scolaire, ce qui l'enchantait grandement.

Bref, ce sont de nombreux commentaires très agréables à recevoir sur notre mobilisation autour du groupe «Stratégies Plus ». Ils contribuent à adoucir les défis que pose ce type de dispositif au quotidien et à réactualiser notre engagement. Un tel projet ne peut être vécu sans la contribution d'une équipe d'intervenants scolaires du tonnerre qui travaille sans relâche pour le mettre en œuvre et le rendre pérenne. Pour nous, ce projet reste une véritable réussite. 